\title{
Using Call/Exit Analysis for Logic Program Transformation
}

\author{
Dmitri Boulanger ${ }^{\star} \quad$ Maurice Bruynooghe ${ }^{\star \star}$ \\ Department of Computer Science, Katholieke Universiteit Leuven \\ Celestijnenlaan 200 A, B-3001, Heverlee, Belgium \\ email: \{dmitri, maurice\}@cs.kuleuven.ac.be
}

\begin{abstract}
A technique for transformation of definite logic programs is presented. A first phase performs an analysis of the extended call/exit patterns of the source program. It is shown that a particular form of correct abstract call/exit patterns can be used as a guide to control the transformation itself and can help to generate the target program having desired properties. The technique provides a framework which, combined with problem specific information concerning the source program, can lead to nontrivial transformations.
\end{abstract}

\section{Introduction}

The most popular approaches for definite logic program transformations are based on unfold/fold and goal replacement operations applied to the clauses of a source program. The unfold/fold transformations have been introduced in [5] and later were applied to logic programming in $[25,15,24]$. The recent paper $[13]^{3}$ revisits the framework developed by Tamaki and Sato.

In this paper we are developing another approach to logic program transformation. Namely, we consider transformations which are performed into two phases: a first phase performs a complete static data flow analysis to derive a transformation guide of the source program, the second phase follows the guide to transform the source logic program (here we follow some basic ideas of $[14,12]$ ). In $[1,2]$ we have introduced a rather general and powerful approach to derive unfold/fold transformations of definite logic programs by abstract interpretation. The unfold/fold transformations, which can be obtained in this way, have been shown to be more general than those of Tamaki and Sato. Indeed, as it was pointed out in $[18,13]$, SLD-like tree analysis can produce transformations which cannot always be expressed in terms of unfold/fold transformations as described in $[25,15,24]$.

* Supported by the K.U.Leuven. Permanent address: Keldysh Institute for Applied Mathematics, Russian Academy of Science, Miusskaya sq., 4, 125047 Moscow, Russia.

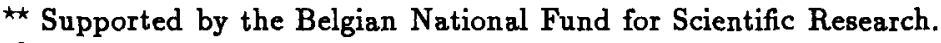

S The examples presented in this paper show that the approach of Tamaki and Sato is not sufficiently sound. 
The paper aims at introducing a logic program transformation framework, which is capable of performing complex transformations by applying unfold/fold and goal replacement transformations which are specialised by problem specific information which are specialised . It is clear, that a toolkit having such a rich set of elementary operations has to be controlled by high level specifications, which are to be sufficiently expressive to describe the target of the transformation of the source program. In this respect the central idea of our approach is to specify the behaviour of the target program in the form of its possible call/exit patterns. The source program can have a lot of different sets of call/exit patterns (by choosing different computation rules), so it is possible to choose the most suitable ones and to obtain by transformation the program, having the specified call/exit patterns, i.e. to fix by transformation the desired behaviour of the program.

The paper is organised as follows: after some preliminaries in section 3 we introduce a special variant of SLD resolution, which is used as basis for our approach. Afterwards we present a technique to represent call/exit patterns and use them as a guide during execution of the definite logic program. The results of the execution are used to transform the program. The new program reflects the specified call/exit structure. In section 4 we give an extensive example, which shows an advanced technique usable for deriving non trivial transformations.

\section{Preliminaries}

In what follows the standard terminology and the basic knowledge of the theory of logic programming, as can be found in [20], is assumed. We will use the standard notions of SLD derivation and refutation, unification and idempotent substitution. The properties of unification and substitutions presented in $[9,19]$ are used indirectly throughout the paper.

The capital letters $B, Q$ and $R$ denote conjunctions of atoms. In the sequel they are considered as collections and are called blocks. The letters $A$ and $H$ denote atoms. Where convenient, we write $E(\bar{X})$ to denote a syntactical object $E$ (block, atom, goal or other expression over the set of terms) with variables $\bar{X}$. By $\operatorname{var}(E)$ we denote the set of variables, occurring in the syntactical object $E($ so $\operatorname{var}(E(\bar{X}))=\bar{X})$.

The greek letters $\theta, \sigma, \varphi$ and $\psi$ will be used to denote idempotent substitutions. Given a substitution $\theta,\left.\theta\right|_{\operatorname{var}(E)}$ will denote the restriction of the substitution $\theta$ to the variables occurring in $E$. Given an expression $E$ over the set of terms, $E \theta$, the instance of $E$ by $\theta$, is defined as usual.

The greek letter $\chi$ is used to denote set of constraints or equations. The most general unifier of a solvable set of constraints is an idempotent substitution $\sigma$ which we denote by $\operatorname{solv}(\chi)$. Given the well known correspondence between solved form and idempotent substitutions [19], we sometimes write $e q(\sigma)$ to denote the solved form corresponding to a substitution $\sigma$. With $\chi$ a set of constraints, by $\chi \sigma$ we mean $\chi \cup e q(\sigma)$. An empty set of constraints $\emptyset$ corresponds to the identity substitution \{\} . We consider a definite $\operatorname{logic}$ program $P$ to be a set of definite clauses $\left\{c_{1}, \ldots, c_{n}\right\}$ equipped with an initial goal $G_{0}$. The succes- 
sive goals in a SLD derivation for the initial goal wrt $P$ will be denoted by $G_{i}$, $i=0,1, \ldots, n$. In the context of this paper we will always deal with programs having exactly the same initial goal $G_{0}=\leftarrow \varepsilon$, where the predicate symbol $\varepsilon$ never appears in the bodies of the clauses $\left\{c_{1}, \ldots, c_{n}\right\}$. The predicate $\varepsilon$ is used to declare the "entry points" of a program. Moreover, given two logic programs $P_{1}$ and $P_{2}$, the intersection of the corresponding languages includes all function symbols but only one predicate symbol, namely $\varepsilon$. It is clear, that all these restrictions cannot influence the generality of the presentation. Finally, we have a simple notion of equivalence of programs: two programs $P_{1}$ and $P_{2}$ are equivalent iff they are logically equivalent wrt the common language (an extensive discussion of the topic can be found in [21]). This notion of logic program equivalence is the most appropriate one for a wide class of applications.

\section{Using Extended Call/Exit Patterns for Controlling Logic Program Transformation}

In this section we present an algorithm for logic program transformation based upon call/exit analysis of an SLD-like tree of the source program $\boldsymbol{P}$. So firstly we describe a special variant of SLD-resolution to be used as the main engine for the call/exit analysis.

\subsection{Extended OLD Resolution}

Extended OLD resolution was introduced by us in $[1,2]$ and deviates from the standard SLD resolution only by imposing certain restrictions on the computation rule. Namely, the SLD derivation

$$
G_{0} \stackrel{c_{1} \theta_{1}}{\longrightarrow} G_{1}, \ldots, \stackrel{c_{n} \theta_{n}}{\longrightarrow} G_{n}
$$

is an EOLD derivation for the initial goal $G_{0}$ wrt a definite logic program $P$ provided that any goal $G_{i}$ in the derivation is represented as an ordered sequences $\left.<B_{1}, B_{2}, \ldots, B_{m}\right\rangle, m \geq 0$ of conjunctions of atoms (blocks) and the computation rule always selects an atom from the first block. Given a goal $G$ represented as an ordered sequence of blocks, $\|G\|$ will denote the collection of all atoms occurring in $G$ (In OLD resolution $[26,16]$ blocks have only one atom).

The following definition gives a more precise description of an EOLD resolution step.

\section{Definition 1. EOLD Resolvent}

Let $G_{i}$ be a goal represented as $\left\langle B_{1}, B_{2}, \ldots, B_{m}\right\rangle, m \geq 1, A$ the atom selected from $B_{1}, c_{i+1}$ a standardised apart input clause from $P, \theta_{i+1}$ the $m g u$ of $A$ and the head of the clause $c_{i+1}$. Then $G_{i+1}$ is derived from $G_{i}$ and $c_{i+1}$ using $m g u$ $\theta_{i+1}$ via the EOLD computation rule if $G_{i+1}$ is obtained by applying the following two steps: 
- construct the auxiliary goal $G^{\prime}=\left\langle B_{1}^{\prime}, B_{2}, \ldots, B_{m}\right\rangle$, where $B_{1}^{\prime}=B_{1}$ with $A$ replaced by the possibly empty body of $c_{i+1}$

- if $B_{1}^{\prime}$ is empty, then $G_{i+1}=\left\langle B_{2}, \ldots, B_{m}>\theta_{i+1}\right.$; else partition the first block $B_{1}^{\prime}$ of $G^{\prime}$ into a number of non empty parts (blocks) $B_{11}^{\prime}, \ldots, B_{1 k}^{\prime}$, $k \geq 1$, and let $G_{i+1}=<B_{11}^{\prime}, \ldots, B_{1 k}^{\prime}, B_{2}, \ldots, B_{m}>\theta_{i+1}$ blocks

In what follows the definition of a subrefutation in an EOLD derivation will play an important role.

\section{Definition 2. EOLD Subrefutation}

An EOLD subderivation of the form $G_{i} \stackrel{c_{i+1} \theta_{i+1}}{\longrightarrow} G_{i+1}, \ldots, \stackrel{c_{n} \theta_{n}}{\longrightarrow} G_{n}$ is an EOLD subrefutation for the first block $B_{1}$ of the goal $G_{i}=\left\langle B_{1}, B_{2}, \ldots, B_{k}\right\rangle, k \geq 1$ if either $G_{n}=<>$ and $k=1$ (i.e. the subderivation is a refutation for $B_{1}$ ) or there exists a substitution $\sigma^{4}$, such that $\left\|<B_{2}, \ldots B_{k}>\sigma\right\|=\left\|G_{n}\right\|$ and there is no goal $G_{j}, i \leq j<n$ having this property in the subderivation. The substitution $\theta_{i+1} \theta_{i+2} \ldots \theta_{n}$ is called an answer substitution of the subrefutation.

Notice that EOLD resolution is sound and complete because soundness and completeness of SLD resolution is independent from the computation rule [20].

Any expression identical, up to variable renaming, with the first block $B$ of the goal $G$ is said to be an extended call of the goal (it will be denoted by call $(G)$ ). The corresponding extended answer (it will be denoted by answ $(G)$ ) is any expression identical, up to variable renaming, with $B \theta$, where $\theta$ is the answer substitution of some refutation of block $B$. Extended call/exit patterns of EOLD trees describe important properties of programs. So below we present a technique for safely approximating the complete set of call/exit patterns.

\subsection{A Safe Approximation of Call/Exit Patterns}

Consider a complete (and, thus, possibly infinite) EOLD tree for a logic program $P$. The complete Call/Exit cover of the tree is the set of all call/exit pairs of the form $[K \Rightarrow A n s w(K)]$, where the key $K$ is an extended call and the set $A n s w(K)$ is the set of all corresponding extended answers. In general the complete $C E$ cover is infinite. So we need a finite abstraction which is a safe approximation. A finite correct abstract $C E$-cover for an EOLD tree can be defined as follows. Suppose there is some equivalence relation over the set of all blocks such that the set of distinct equivalence classes is finite. Then the set of keys occurring in the complete $C E$-cover can be represented by a finite set $\left\{K_{1}^{\sim}, K_{2}^{\sim}, \ldots, K_{n}^{\sim}\right\}$ of equivalence classes. Let $A n s w^{\sim}\left(K^{\sim}\right)$ be the finite set of equivalence classes of blocks occurring in a set

$$
\bigcup_{K \in K^{\sim}} A n s w(K)
$$

4 In the context of EOLD resolution the substitution $\sigma$ is exactly the substitution $\theta_{i+1} \ldots \theta_{n}$. Below we will use the modified EOLD resolution, where this property is not always the case. 
Then the complete abstract $C E$-cover is a finite set of abstract call/exit pairs

$$
\left\{\left[K_{i}^{\sim} \Rightarrow A n s w^{\sim}\left(K_{i}^{\sim}\right)\right] \mid i=1, \ldots, n\right\} .
$$

Let $G$ be a goal occurring in an EOLD tree. Given a complete abstract $C E$-cover, the extended call call $(G)$ is said to be covered by the key $K^{\sim}$ iff $\operatorname{call}(G) \in K^{\sim}$. Similarly, the extended answer answ $(G)$ is said to be covered iff the corresponding extended call call $(G)$ is covered by the key $K^{\sim}$ and answ $(G)$ belongs to some equivalence class occurring in the set $A n s w^{\sim}\left(K^{\sim}\right)$.

The complete abstract cover requires all call/exit pairs to be covered. In the sequel we will need a more flexible description of program properties: we will allow that not all call/exit pairs are covered. This will be used to control the transformation of the source program $P$ (see below). Consider the following more flexible description of call/exit patterns.

Definition 3. Safe Abstract Call/Exit Cover

Given an EOLD tree, a safe abstract $C E$-cover of the tree is a subset of the complete abstract finite $C E$-cover such that the number of not covered extended calls and answers occurring in the EOLD tree is finite.

In what follows we will always assume that the EOLD tree and the corresponding safe abstract $C E$-cover satisfy the following conditions: ${ }^{5}$

- Every equivalence class can be represented by a block of atoms. All elements of an equivalence class are instances of its corresponding block. Thus we will not distinguish between equivalence classes and their corresponding blocks.

- The block size of an EOLD tree is bounded by some constant.

The conditions above ensure that any EOLD tree has a (safe) abstract finite $C E$-cover. For example, a trivial $C E$-cover can be obtained using only recursive predicate symbols of the program $P$ with distinct variables as arguments to construct the blocks of the equivalence classes.

Example 1. The empty set is a safe abstract $C E$-cover for any EOLD tree, which can be constructed for any logic program $P$ having a finite extended minimal model $E M M_{P}$ (see [10]) provided that the block size is bounded. Also the set

$$
\left\{\left[q(\bar{X}) \Rightarrow E M M_{P}(q)\right] \mid q \in P\right\}
$$

is always a safe (possibly infinite) $C E$-cover for any program $P$, where $q \in P$ are the predicates of $P$ and $E M M_{P}(q) \subseteq E M M_{P}$ are the elements of the extended minimal model of $P$ having a predicate symbol $q$. Moreover it is complete $C E$ cover.

On the other hand, the empty set is not a safe cover of any OLD tree, which can be constructed for the program

$$
\begin{aligned}
& \varepsilon(X) \leftarrow \operatorname{even}(s(X)) . \\
& \text { even }(0) . \\
& \text { even }(s(s(X))) \leftarrow \operatorname{even}(X) .
\end{aligned}
$$

${ }^{b}$ These conditions are not strictly necessary. We use them to simplify the presentation. 
with the standard goal $\leftarrow \varepsilon(X)$, because the number of not covered answers is infinite. Notice, that the number of not covered calls is finite. The abstract $C E$-cover

$$
\{[\operatorname{even}(X) \Rightarrow\{\operatorname{even}(Y)\}]\}
$$

is safe for any OLD tree, which can be constructed for the above program.

A safe abstract $C E$-cover can be used to control EOLD resolution and to construct a tree having a special shape.

\subsection{Modified EOLD Resolution}

Our framework uses a special variant of EOLD resolution. In order to introduce it we need some auxiliary definitions. Firstly, we will use extended goals, which are sequences of blocks and exit markers. An exit marker is a block which is syntactically different from any block of atoms and has the form $\triangle_{G}(\sigma, \chi)$, where $G$ is a goal in the current EOLD derivation, $\sigma$ is a substitution and $\chi$ is a set of constraints. The goal $G$ is the goal, where the exit marker has been inserted. The initial goal always has the form $G_{0}=\left\langle\varepsilon, \Delta_{G_{0}}(\{\}, \emptyset)\right\rangle$. Given a substitution $\theta$, we will assume that

$$
\triangle_{G}(\sigma, \chi) \theta=\triangle_{G}(\sigma, \chi \cup e q(\theta)) \text {. }
$$

Secondly, we will use two special operations: a key factorisation and an exit marker elimination. The former inserts an exit marker, while the latter deletes it. Key factorisation is a transformation of a goal $G=\left\langle B_{1}, B_{2}, \cdots, B_{n}\right\rangle$, where $B_{1}$ is a block of atoms. If there exists a standardised apart key $K=K(\bar{W})$ in the $C E$-cover, i.e. $\operatorname{var}(K)=\bar{W}, \bar{W} \cap \operatorname{var}(G)=\emptyset$, and a substitution $\sigma$, $\operatorname{dom}(\sigma)=\bar{W}$ such that $B_{1}=K \sigma$ (i.e. the block $B_{1}$ is covered by the key $K$ ), then the key factorisation replaces the goal $G$ by $\left\langle K, \triangle_{G}(\sigma, \emptyset), B_{2}, \cdots, B_{n}\right\rangle$. It is important to notice that after key factorisation the first block of the goal has "fresh" variables, which differ from the variables in the other blocks of atoms.

The exit marker elimination is applicable to a non singleton goal if the goal has at least two exit markers and if its first element is an exit marker. Let

$$
G=<\triangle_{G_{1}}\left(\sigma_{1}, \chi_{1}\right), B_{1}, \ldots, B_{m}, \triangle_{G_{2}}\left(\sigma_{2}, \chi_{2}\right), \cdots>, m \geq 0
$$

be a goal, where $\triangle_{G_{2}}\left(\sigma_{2}, \chi_{2}\right)$ is the second exit marker in the goal. There exists always at least one exit maker because any goal contains the exit marker of the initial goal, i.e. any goal has the form $\left\langle\cdots, \Delta_{G_{0}}(\{\}, \chi)\right\rangle$. Suppose that $\theta=\operatorname{solv}\left(\chi_{1}\right) \neq$ fail and $G_{1}=\left\langle K(\bar{W}), \Delta_{G_{1}}\left(\sigma_{1}, \emptyset\right), \cdots>\right.$ with $\operatorname{dom}(\sigma)=\bar{W}$ (cf. key factorisation above). Then the safety of the $C E$-cover ensures (see def. 4 below, which describes the structure of a derivation), that there exist a key $K=K(\bar{W})$ and a corresponding answer $K \sigma_{\text {answ }}$ with $\operatorname{dom}\left(\sigma_{\text {answ }}\right)=\bar{W}$ such that $K \theta$ is an instance of $K \sigma_{a n s w}$, i.e.

$$
K \theta=\left(K \sigma_{a n s w}\right) \varphi, \text { and } \operatorname{solv}\left(e q\left(\sigma_{a n s w}\right) \cup e q(\varphi)\right)=\operatorname{solv}\left(\chi_{1}\right)
$$

Then the exit marker elimination creates the new goal $G^{\prime}=\operatorname{exit}(G)$,

$$
G^{\prime}=\left\langle B_{1}, \ldots, B_{m}, \Delta_{G_{2}}\left(\sigma_{2}, \chi_{2} \cup e q(\varphi)\right) \cdots>\psi,\right.
$$


where $\psi=\operatorname{solv}\left(e q\left(\sigma_{1}\right) \cup e q\left(\sigma_{a n s w}\right)\right)$. The substitution $\psi$ is exactly the part of the answer which is "prescribed" by the $C E$-cover to instantiate the remaining part of the goal. Therefore, $\psi$ is applied to the whole goal, while the not allowed part $\varphi$ is added to the constraints of the next exit marker. In this way $\varphi$ is isolated (but not lost!) in the next exit marker. This explains why it is always necessary to have at least one exit marker - it accumulates the parts of the answers which have to be delayed.

The extensions above allow to modify standard EOLD resolution as follows. Let us denote by resolve $E O L D(G, c)$ the goal, which can be derived from the goal $G$ and input clause $c \in P$ by applying a standard EOLD resolution step (cf. def. 1).

Definition 4. (Modified) EOLD* Resolvent

Given a $C E$-cover and an extended goal $G=\left\langle B_{1}, B_{2}, \cdots, B_{n}\right\rangle$, the (modified) EOLD* resolution step

$$
G_{i} \stackrel{\theta_{i+1}}{\longrightarrow} G_{i+1}, G_{i+1}=\operatorname{resolve}_{E O L D}^{*}\left(G_{i}, c_{i+1}\right)
$$

consists of one of the following operations:

1. If $B_{1}$ is a block of atoms (not an exit marker), derive the intermediate goal

$$
G^{\prime}=\operatorname{resolve}_{E O L D}\left(G_{i}, c_{i+1}\right)
$$

and construct the corresponding substitution $\theta_{i+1}$ and:

If the goal $G^{\prime}$ has the form $\left\langle B_{1}^{\prime}, B_{2}^{\prime}, \cdots\right\rangle$, where $B_{1}^{\prime}$ is a block of atoms such that the key factorisation on $B_{1}^{\prime}$ is applicable,

then $G_{i+1}=\left\langle K, \Delta_{G_{i+1}}(\sigma, \emptyset), B_{2}^{\prime}, \cdots>\right.$

else (the key factorisation is not applicable) $G_{i+1}=G^{\prime}$.

2. If the goal $G$ has the form $\left\langle\Delta_{G}(\sigma, \chi), \cdots\right\rangle$ (the first block is an exit marker) do:

if the exit marker elimination is applicable on $\triangle_{G}(\sigma, \chi)$

then $G_{i+1}=\operatorname{exit}\left(G_{i}\right), \theta_{i+1}=\sigma$

else (exit marker elimination is not applicable) the goal is non-extendable

In the sequel we will assume that all failed branches of the modified EOLD* tree are dropped. A branch of the tree is considered to be a failed branch iff it contains a non extendable goal $G$ such that it is either a failed goal in standard sense (cf. def. 1) or the set of all constraints occurring in $G$ is not solvable. Then definition 4 above ensures the following simple properties of the modified EOLD* trees:

- A goal $G$ is a non extendable goal iff it is a success goal of the form $G=<$ $\triangle_{G_{0}}(\{\}, \chi)>$, solv $(\chi) \neq$ fail. 
- The definition of subrefutation (cf. def. 2) and the notions of extended calls and answers, which have been introduced above in the context of standard EOLD resolution (cf. section 3.1), are also applicable to the goals of the modified EOLD* trees (including the goals having an exit marker as a first element), but the answer substitutions have another meaning (see prop. 5 and 6 below).

- Each covered extended call occurring in the tree occurs as a key in the $C E$ cover

Consider a standard EOLD tree and the corresponding modified EOLD* tree constructed wrt some safe finite abstract $C E$-cover. The relation between them is given in the following proposition:

Proposition 5. The EOLD* tree constructed using some safe finite abstract $C E$-cover contains a derivation

$$
G_{0} \stackrel{\theta_{1}}{\longrightarrow} G_{1}, \ldots, \stackrel{\theta_{n}}{\longrightarrow} G_{n}
$$

having the final success goal $G_{n}$ of the form $\left\langle\Delta_{G_{0}}(\{\}, \chi)\right\rangle, \sigma=\operatorname{solv}(\chi) \neq$ fail (i.e. the refutation of the initial goal) iff the standard EOLD tree contains a refutation

$$
G_{0} \stackrel{\theta_{1}^{\prime}}{\longrightarrow} G_{1}^{\prime}, \ldots, \stackrel{\theta_{m}^{\prime}}{\longrightarrow} G_{m}^{\prime}, n \geq m
$$

such that $G_{n}^{\prime}=<>$ and $\operatorname{solv}\left(e q\left(\theta_{1}\right) \cup \ldots \cup e q\left(\theta_{n}\right) \cup \chi\right)=\operatorname{solv}(\chi)=\theta_{1}^{\prime} \ldots \theta_{m}^{\prime}$.

The following proposition describes the most important feature of EOLD* resolution.

Proposition6. Given an EOLD* tree constructed using some safe finite abstract $C E$-cover, any extended call of an extendable goal occurring in the tree has a finite number of corresponding extended answers, while the length of the corresponding subrefutation and their total number can be unbounded.

The propositions 5 and 6 are very important for the transformation algorithm introduced below.

\subsection{Transformation Algorithm}

Given an EOLD* tree constructed wrt some safe abstract $C E$-cover, the algorithm for generating a new logic program $I$ is the following:

1. Introducing New Predicate Symbols: for each key $K$ different from $\varepsilon$ in the $C E$-cover generate a fresh predicate symbol $\pi / n$, where $n$ is the number of distinct variables occurring in the key $K^{6}$. The predicate symbol $\varepsilon$ is also included in the set of predicate symbols of the program $I I$. In this way each covered extended call in the EOLD* tree is associated with a new predicate symbol of $I I$.

- In [3] we give powerful conditions, which allow for dropping some arguments of the new predicates. 
2. Synthesising New Program Clauses: for each subrefutation of the first block of a goal $G$ having the form

$$
G \stackrel{\theta_{1}}{\longrightarrow} G_{1}, \ldots, \stackrel{\theta_{n}}{\longrightarrow} G_{n}
$$

where $G=\left\langle K, \Delta_{G}(\sigma, \emptyset), \cdots\right\rangle$ and $G_{n}=\left\langle\Delta_{G}(\sigma, \chi), \cdots\right\rangle$ (i.e. the key factorisation has been successful for the goal $G$ ), construct a new clause of the program $I$ of the form

$$
\left(\pi_{0}\left(\bar{X}_{0}\right) \leftarrow \pi_{i_{1}}\left(\bar{Y}_{i_{1}}\right), \ldots, \pi_{i_{m}}\left(\bar{Y}_{i_{m}}\right)\right) \operatorname{solv}(\Sigma)
$$

where:

- $\pi_{0}$ is a new predicate symbol associated with the block of atoms $B_{0}$ (covered extended call of $G$ ) and $\bar{X}_{0}=\operatorname{var}\left(B_{0}\right)$

- $G_{i_{j}} \neq G, j=1, \ldots, m$ are all goals occurring in the subrefutation $B_{0}$ of the form

$$
G_{i_{j}}=<B_{i_{j}}, \Delta_{G_{i_{j}}}\left(\sigma_{i_{j}}, \chi_{i_{j}}\right), \cdots, \Delta_{G}(\cdots) \cdots>\text {, }
$$

such that the exit marker $\Delta_{G}\left(\sigma_{i_{j}}, \chi_{i_{j}}\right)$ is exactly the second element in the goal (the latter means that we consider only the top level covered extended calls occurring in the subrefutation of $B_{0}$ )

- $\pi_{i j}$ is the predicate associated with the covered extended call of $G_{i_{j}}$ (which corresponds to the block of atoms $B_{i_{j}}$ ) and $\bar{Y}_{i_{j}}=\operatorname{var}\left(B_{i_{j}}\right)$, $j=1, \ldots, m$

- $\Sigma=\bigcup_{k} e q\left(\theta_{i_{h}}\right)$, where $\theta_{i_{k}}$ is the substitution labelling the EOLD* step

$$
G_{i_{h}-1} \stackrel{\theta_{i_{h}}}{\longrightarrow} G_{i_{h}}
$$

and the goals $G_{i_{k}} \neq G, k=1, \ldots, l$ are all the goals in the subrefutation of $B_{0}$ having the form

$$
G_{i_{h}}=<B s_{i_{h}}, \Delta_{G}\left(\sigma, \chi_{i_{h}}\right), \cdots>
$$

or

$$
G_{i_{h}}=<B_{i_{h}}, \Delta_{G_{i_{h}}}\left(\sigma_{i_{h}}, \chi_{i_{h}}\right), \cdots, \Delta_{G}(\cdots) \cdots>
$$

where $B s_{i_{k}}$ is a (possibly empty) sequence of blocks of atoms (here we consider all top level calls occurring in the subrefutation of $B_{0}$ )

For the clauses which are synthesised from the refutations of the initial goal $G_{0}=<\varepsilon, \Delta_{G_{0}}(\{\}, \emptyset)>$ the predicate $\varepsilon$ is used to construct the clause head. Notice that $\bar{X}_{0}, \bar{Y}_{i_{j}}, j=1, \ldots, m$ are disjoint sets of variables.

3. The Final Program: consider the set of all clauses, which can be obtained following the algorithm above. If clauses which are renaming of each other are considered identical, then the set of clauses is finite. Moreover, the new program $I$ is logically equivalent to the source program $\boldsymbol{P}$ wrt the common language, which consists of only one predicate symbol $\varepsilon$ and all function symbols. 
To be convinced that the algorithm always generates a finite program, it is sufficient to notice the following:

- All goals of the form $G=\left\langle K, \Delta_{G}(\cdots) \cdots\right\rangle$ have exactly the same (up to the variable renaming) set of subrefutations for the first block, because the key factorisation always creates the first block with fresh variables, and thus the set of subrefutations is independent from the particular goal in the tree. In other words, the influence of the other blocks on the goal is isolated (delayed) in the exit marker in the form of a set of constraints. This means, that the final program has a finite number of predicate definitions (one for each key in the $C E$-cover and one for $\varepsilon$ ).

- The number of clauses in each definition is finite. Suppose that there is a definition having an infinite number of clauses. This implies, that the extended call, which corresponds to the predicate symbol of the definition, has an infinite number of extended answers. This contradicts proposition 6. For the same reason the number of atoms in the bodies of the clauses is also bounded.

The equivalence of the source program $P$ and the program $\Pi$ can be derived by using proposition 5 and by showing that any EOLD* refutation of $P$, which is constructed wrt a chosen safe finite $C E$-cover, can be simulated by some OLD* refutation (EOLD ${ }^{*}$ with singleton blocks!) of $\Pi^{7}$, which is constructed wrt the trivial $C E$-cover (constructed from the new predicates having distinct variables as arguments) and vice versa.

Let us provide some important remarks concerning the applicability of the logic program transformation framework, which has been introduced above. The presentation above was given under assumption that there exists an EOLD tree having a safe $C E$-cover. Finding the EOLD tree and the $C E$-cover is intended to be solved separately during a first phase. In the next section we will give several remarks concerning this problem.

It should be clear from the discussion above, that we really need to construct only some upper portion of the EOLD* tree. Namely, all keys occurring in the $C E$-cover and some portion of the corresponding subrefutations have to be discovered in the tree ${ }^{8}$. This problem is also addressed in the next section.

The presentation itself of the transformation algorithm was given in a form, which was as simple as possible (the price of the simplicity is that the algorithm uses too many intermediate variables, which are not always necessary). For the same reason a tabulation mechanism [26]) was not included too. Here we were mainly interested in the careful investigation of the class of logic program transformations (see sections 4 and 5 below), which can be derived in our framework. So we have omitted technical details related to an efficient implementation of the

7 This means, that the algorithm is capable to derive FOLD transformations.

- Using the specially constructed $C E$-cover we significantly extend the "upper portion" algorithms of $[22,23]$. For example, in this way we avoid Eureka-steps when generating complex folding transformations (see also $[1,2]$ ). 
algorithm (a variant of the algorithm. which is rather efficient from an implementation point of view, but has a more narrow class of derivable transformations can be found in $[1,2])$.

Finally, notice, the great importance of the particular structure of the chosen safe abstract $C E$-cover. It can be used to control the structure of the new program: the keys specify the granularity of the definitions of $\Pi$, while the answers can be used to control the internal structure of the definitions and the structure of the clauses of $I I$. By allowing some extended calls and answers not to be covered (cf. example 1), we can specify what should be factored out by partial evaluation: all procedure calls not corresponding to the keys in the $C E$-cover are partially evaluated (cf. [18]). Thus, the particular form of the equivalence relation over the set of blocks is crucial. It seems that, by choosing non trivial equivalence relations, one can obtain rather complex and deep transformations ${ }^{9}$.

\section{Deriving Logic Programs Using Call/Exit Patterns of the Source Program}

As could be seen from above the EOLD* interpretation and subsequent transformation algorithm are strictly controlled by the safe abstract $C E$-cover of some EOLD tree. So the first problem is to prove the existence of the desired EOLD tree and to find a safe approximation of its call/exit patterns. This problem is well known and several frameworks based on abstract top-down computation equipped with the tabulation mechanism have been suggested $[26,16,7]$. It was shown, that abstract tabled computations can be done correctly for a very wide class of abstract domains $[16,7]$. These algorithms allow to construct complete finite abstract $C E$-covers of some OLD tree using some equivalence relation over the set of atoms. Definition 1 has been elaborated keeping in mind the applicability of these algorithms to the extended call/exit patterns, which are used in our framework. Thus, we can assume, that we are given some equivalence relation $\mathcal{R}$ and the corresponding complete abstract $C E$-cover $\mathcal{C E}(\mathcal{R})$. Notice, that $\mathcal{C E}(\mathcal{R})$ can imply a particular blocking strategy of some EOLD tree, i.e. the rule for partitioning atoms of the current goal into blocks (cf. def. 1).

Below we provide an example of a program transformation, which uses a more general form of $\mathcal{R}$ than was described in section 3.1. This example is intended to demonstrate the capabilities of the framework and the usefulness of nontrivial equivalence relations over the set of atoms.

Consider the logic program $P$ given in example 1. The abstract $C E$-cover

$$
\{[\operatorname{even}(s(X)) \Rightarrow\{\operatorname{even}(s(s(0))),<\operatorname{even}(s(s(Y))), Y \neq 0>\}]\}
$$

is not a safe abstract $C E$-cover for $P$, because the not covered call even $(X)$ has an infinite number of exit patterns. Some modification of the framework presented in section 3 allows to derive a finite new program. The OLD* tree

\footnotetext{
In [3] we use an equivalence relation over the set of blocks, which considers two blocks to be equivalent if they are equivalent goals wrt the source program $P$.
} 
contains the following simple derivation

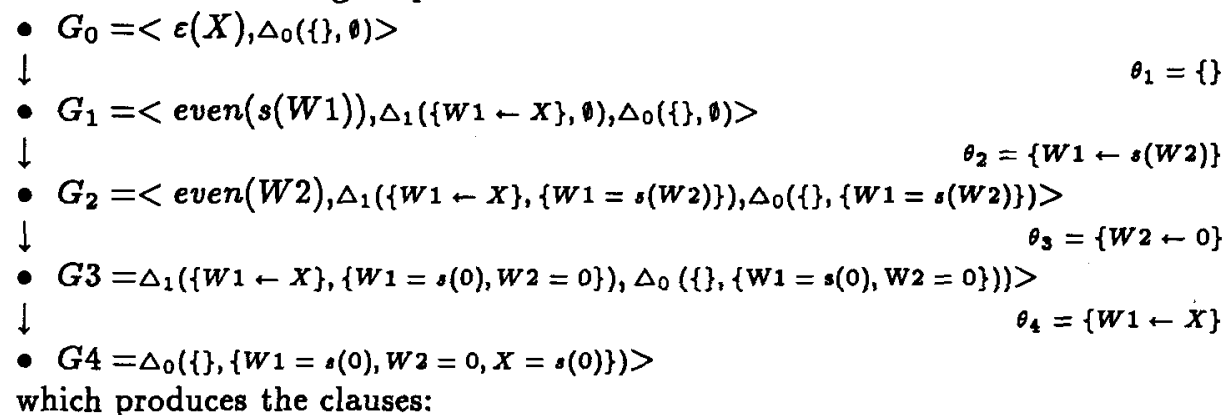

- the clause $\varepsilon(X) \leftarrow \pi_{1}(X)$, which was obtained from the subrefutation of $\operatorname{call}\left(G_{0}\right)$ by

$$
\left(\varepsilon(X) \leftarrow \pi_{1}(W 1)\right) \operatorname{solv}\left(e q\left(\theta_{1}\right) \cup e q\left(\theta_{4}\right)\right)
$$

- the clause $\pi_{1}(s(0))$, which was obtained from the subrefutation of $\operatorname{call}\left(G_{1}\right)$ by

$$
\left(\pi_{1}(W 1)\right) \operatorname{solv}\left(e q\left(\theta_{2}\right) \cup e q\left(\theta_{3}\right)\right)
$$

The subrefutations for $\operatorname{call}\left(G_{1}\right)$ of the form $G_{1} \stackrel{\theta_{2}}{\longrightarrow} G_{2} \stackrel{\theta_{\mathrm{s}}}{\longrightarrow} G_{5} \longrightarrow \cdots$, where $\theta_{\mathrm{b}}=\{W 2 \leftarrow s(s(W 3))\}$ and

$G_{5}=\left\langle\operatorname{even}(W 3), \Delta_{1}\left(\left\{W_{1} \leftarrow X\right\},\left\{W_{1}=s(s(s(W 3))), W_{2}=s(s(W 3))\right\}\right), \Delta_{0}(\{\},\{\cdots\})>\right.$ will produce an infinite number of clauges $\pi_{1}(s(s(s(0)))), \pi_{1}(s(s(s(s(s(0)))))), \cdots$, if the algorithm from section 3.4 is used.

Thus we have to control the uncovered exit patterns of the call even $(X)$ "manually". We can apply apply some "massaging" on $G_{5}$ such that we avoid the the infinite number of distinct subrefutations for even(W3). It can be done as follows. The substitution $\theta_{5}$ is the first one, which creates an "incomplete" but already not allowed answer for call $\left(G_{1}\right)$. Thus $\theta_{5}$ has to be made more flat. In general it is a complex operation, which is not always possible (because we should preserve correctness of all refutations in the tree), but in this particular case the following reconstruction is correct: $\theta_{5}^{\prime}=\left\{W_{2}-s\left(W_{4}\right)\right\}$ and $G_{5}^{\prime}=<\operatorname{even}\left(s(W 4), \Delta_{1}(\{W 1 \leftarrow X\},\{W 1=s(s(W 4)), W 2=s(W 4)\}), \Delta_{0}(\{\},\{\cdots\})>\right.$, Now key factorisation on $G_{5}^{\prime}$ is applicable! One obtains: $G_{5}^{\prime \prime}=$ $<\operatorname{even}\left(s(W 5), \Delta_{b}(\{W 5 \leftarrow W 4\}, \emptyset), \Delta_{1}(\{W 1 \leftarrow X\},\{W 1=s(s(W 4)), W 2=s(W 4)\})\right.$, $\Delta_{0}(\{\},\{\cdots\})>$.

The reconstruction above is ensured by the available abstract $C E$-cover. Indeed, the variable $W 3$ occurs in only one atom of the goal $G_{5}$ and it can possibly be instantiated by resolving even $(W 3)$. On the other hand, the $C E$-cover ensures, that the new introduced variable $W 4$ occurring in $G_{5}^{\prime}$ can be instantiated only by $s(U)$ when resolving even $(s(W 4))$ in $G_{5}^{\prime}$ and thus, the variable $W 2$ will have the same value as in the original derivation, provided that all constraints in $G_{5}^{\prime}$ are modified accordingly. This reconstruction can be considered as applying a special equivalence relation over the set of atoms, which can be defined wrt the source program $P$. On the other hand, this reconstruction is something more 
powerful than the well known goal replacement (see [13, 17, 3]). In [2] it is shown how the formal proof of the equivalence above can be obtained.

The reconstructed subrefutations of the form $G_{1} \stackrel{\theta_{2}}{\longrightarrow} G_{2} \stackrel{\theta_{5}^{\prime}}{\longrightarrow} G_{5}^{\prime \prime} \stackrel{\theta_{5}}{\longrightarrow} G_{6} \cdots$, where $\operatorname{call}\left(G_{6}\right)=\operatorname{even}(W 6)$ and $\theta_{6}=\{W 5 \leftarrow s(W 6)\}$, will produce only one clause $\pi_{1}(s(s(W 4))) \leftarrow \pi_{1}(W 4)$ by

$$
\left(\pi_{1}(W 1) \leftarrow \pi_{1}(W 5)\right) \operatorname{solv}\left(e q\left(\theta_{2}\right) \cup e q\left(\theta_{5}^{\prime}\right) \cup\{W 5=W 4\}\right),
$$

i.e. the following new program is generated

$$
I=\left\{\varepsilon(X) \leftarrow \pi_{1}(X), \quad \pi_{1}(s(0)), \quad \pi_{1}(s(s(X))) \leftarrow \pi_{1}(X)\right\} .
$$

Notice, that it was sufficient to consider only some upper portion of the tree. In general, if a covered call has $s$ answers in the $C E$-cover, then only $s$ derivations, which are passing it should be considered (this can be implemented by applying the corresponding tabulation mechanism). In the case of OLD* resolution by allowing to consider several answers we extend the algorithms of $[11,12]$, where only one answer can be considered. In complex applications similar to meta-programs specialisation multiple answers can help to increase the granularity of residual programs. By using OLD* resolution with $C E$-covers having only singleton sets of answers, our algorithm reduces to that [12]. On the other hand in the example above the specification of the pair of answers was important and was used in another way (otherwise the goal reconstruction would not be possible).

The new program $I$ is the odd numbers program. This kind of transformations cannot be produced using the frameworks of $[11,12,17,22,23]$ (in our case it has been possible due to incorporating of special information about the source program). This example shows that our approach can be used in a problem specific way by introducing specialised equivalence relations. We expect that very interesting transformations can be obtained by using equivalence relations over blocks of unbounded size (the number of equivalence classes should be finite). In this way folding of an infinite number of atoms can be derived (the recent paper [4] gives an example). Equivalence relations of the latter kind can produce transformations similar to $[6,8]$. Extensive examples of standard "finite" folding transformations, which can be obtained by applying a weaker version of the presented algorithm are given in $[1,2]$.

\section{Conclusion and Related Work}

Our framework allows to consider the source logic program as a "runnable" specification, which should be used to derive a "real" program, which satisfies some requirements. Indeed, one can perform the following step:

1. Choose the most suitable abstract domain for the program at hand. The domain should satisfy the necessary conditions for the correct application of an abstract tabulation mechanism (see for example $[16,7]$ ). This step is 
not formal and should take into account all available information about the program and its application (sometimes it can be provided by the users of the program).

2. In terms of the abstract domain specify the desired call/exit patterns of the target program. The following items can be specified:

- What should be factored out by partial evaluation

- The macro structure of the program, i.e. the number of the definitions and their granularity

- The internal structure of the clauses

Prove by applying an abstract tabulation technique, that the specified call/exit patterns imply the existence of an EOLD tree.

3. Apply the algorithms described in sections 3.3 and 3.4.

The basic ideas of the approach allow to integrate in one toolkit lots of existing transformation techniques for definite logic programs $[18,13,11,12$, $22,23]$ combined with high level concepts to specify the properties of the target program and possibilities to incorporate problem specific information. It seems, that the development of a general form for the requirements on the equivalence relations over the set of blocks is a promising direction of research.

\section{References}

1. Boulanger,D., Bruynooghe,M., Deriving Transformations of Logic Programs using Abstract Interpretation, Logic Program Synthesis and Transformation (LOPSTR'92), eds. K.K.Lau and T.Clement, Workshops in Computing, Springer Verlag, 1993, 99-117.

2. Boulanger,D., Bruynooghe,M., Deriving Fold/Unfold Transformations of Logic Programs Using Extended OLDT-based Abstract Interpretation, J. Symbolic Computation, 1993, Vol.15, 495-521.

3. Boulanger,D., Bruynooghe,M., Using Abstract Interpretation for Goal Replacement, Logic Program Synthesis and Transformation LOPSTR'93, Workshops in Computing, Springer-Verlag, 1993.

4. Boulanger,D., De Schreye,D., Compiling Control Revisited: A New Approach based upon Abstract Interpretation, Proc. 11-th Int. Conf. Logic Programming, 1994, 699-713.

5. Burstall,R., Darlington,J., A Transformation System for Developing Recursive Programs, JACM, Jan.1977, Vol.24, No.1, 44-67.

6. Bruynooghe,M., De Schreye,D., Krekels,B., Compiling Control, J. Logic Programming, 1989, Vol.6, Nos.2-3, 135-162.

7. Codognet,P., File, G., Computations, Abstractions and Constrainte in Logic Programs, Proc. $4^{\text {th }}$ Int. Conf. on Programming Languages, Oakland, USA, April 1992.

8. De Schreye,D., Martens,B., Sablon,G., Bruynooghe,M., Compiling Bottom- $U_{p}$ and Mixed Derivations into Top-Down Executable Logic Programs, J. Automated Reasoning, 1991, 337-358.

9. Eder,E., Properties of Substitutions and Unifications, J. Symbolic Computation, 1985, Vol.1, No.1, 31-46. 
10. Falaschi,M., Levi,G., Martelli,M., Palamidessi,C., Declarative Modelling of the Operational Behaviour of Logic Languages, Theoretical Computer Science, 1989, Vol.69, No.3, 289-318.

11. Gallagher,J., Bruynooghe,M., Some Low Level Transformations of Logic Programs, Proc. $2^{\text {nd }}$ Workshop in Meta-Programming in Logic, Leuven, 1990, 229 244.

12. Gallagher,J., Bruynooghe,M., The Derivation of an Algorithm for Program Specialisation, New Generation Computing, 1991, Vol.9, 305-333.

13. Gardner, P., Shepherdson, J., Unfold/Fold Transformations of Logic Programs, Computational Logic, Essays in Honour of Alan Robinson, eds. J.L.Lassez and G.Plotkin, MIT Press, 1991, 565-583.

14. Gallagher, J., Codish M., Shapiro E., Specialisation of Prolog and FCP Programs Using Abstract Interpretation, New Generation Computing, 1988, Vol.6, Nos.2-3, 159-186.

15. Kawamura, T., Kanamori, T., Preservation of Stronger Equivalence in Unfold/Fold Logic Program Transformation, Proc. $4^{\text {th }}$ Int. Conf. on FGCS, Tokyo,1988.

16. Kanamori, T., Kawamura, T., Abstract Interpretation Based on OLDT Resolution, J. Logic Programming, 1993, Vol.15, Nos.1-2, 1-30 .

17. Kawamura, T., Derivation of Efficient Logic Programs by Synthesising New Predicates, Proc. 1991 Int. Symp. on Logic Programming, San Diego, 1991, 611-625.

18. Lloyd,L., Shepherdson,J., Partial Evaluation in Logic Programming, J. Logic Programming, 1991, Vol.11, Nos.3-4, 217-242.

19. Lassez, J.-L., Maher, M., Mariott, K., Unification Revisited, Foundations of Deductive Databases and Logic Programming, ed. J.Minker, Morgan-Kaufmann, 1988, 587-625.

20. Lloyd,L., Foundations of Logic Programming, Springer-Verlag, Berlin, 1987.

21. Maher,M., Equivalences of logic Programs, Foundations of Deductive Databases and Logic Programming, ed. J.Minker, Morgan-Kaufmann, 1988, 627-658.

22. Proietti,M., Pettorossi,A., Construction of Efficient Logic Programs by Loop Absorption and Generalisation, Proc. $2^{\text {nd }}$ Workshop in Meta-Programming in Logic, Leuven, 1990, 57-81.

23. Proietti,M., Pettorossi,A., Unfolding - Definition - Folding, In this Order, For Avoiding Unnecessary Variables in Logic Programs, Proc. $3^{\text {rd }}$ Int. Symp. on Programming Languages Implementation and Logic Programming, Aug. 1991, LNCS No.528, Springer-Verlag, 1991, 347-358.

24. Seki,H., Unfold/Fold Transformation of stratified programs, J. Theoretical Computer Science, 1991, Vol.86, 107-139.

25. Tamaki,H., Sato,T., Unfold/Fold Transformation of Logic Programs, Proc. $2^{\text {nd }}$ International Conference on Logic Programming, Uppsala, 1984, 127-138.

26. Tamaki,H., Sato,T., OLD Resolution with Tabulation, Proc. $3^{\text {rd }}$ Int. Conf. on Logic Programming, London, July 1986, 84-98. 